\title{
HIGH-RESOLUTION GEOMETRY MEASUREMENT WITH A COLLABORATIVE ROBOT
}

\author{
M. Ried ${ }^{1 *}$, R. Danzl ${ }^{1}$, M. Felberbauer ${ }^{1}$, T. Lankmair ${ }^{1}$ \\ ${ }^{1}$ Alicona Imaging GmbH, Raaba, Austria \\ *Corresponding author; e-mail: matthias.riedl@bruker.com
}

\begin{abstract}
The improvements of robust optical 3D metrology and robot systems are opening new possibilities of inline optical geometry measurements. The combination of these two facts allows measurements using the robot as manipulation system to increase the measurement area beyond a single field of view.

By examining the sample from different angles using the robot to obtain high-resolution 3D datasets also from undercuts, measurements which have to date not been possible due to light/shade influences can now be achieved. This method is important on big samples with complex geometries. Special algorithm uses the position and angle of the robot tool centre point, in this case the focus point of the measuring system, for the pre-alignment of the different 3D datasets before the final stitching is performed. Further algorithm is used to improve the overall accuracy through dataset alignment.

In this paper, measurement examples are presented, which examine and aim to minimize the uncertainty of this optical 3D metrology and robot combination and are tested against international standards.
\end{abstract}

\section{Keywords:}

Inline, collaborative, automation, 3D measurement, optical metrology, 3D dataset, integration, robot, Focus Variation

\section{INTRODUCTION}

The ongoing miniaturization of features on industrial parts, the reduction of manufacturing tolerances and the increasing complexity regarding the overall part geometry generates the necessity of highly accurate 3D measurement devices for measurements with sub- $\mu \mathrm{m}$ resolution. Examples for this kind of precise, complex and heavy parts can be found in many variations in the aeronautic industry or also samples which are created by adaptive manufacturing.

These changes lead to an increasing demand for new metrology methods to get more information about the geometric features which are high precision-manufactured.

A well know approach for these kinds of measurements on complex geometries are tactile measuring systems or optical measuring systems based on white light interferometry, structured light, confocal microscopy or Focus-Variation [Weckenmann 2012] [Danzl et al. 2011].

The limits for the tactile systems are given by the geometry of the touch probe and can be bypassed by using different additional systems for the rotation and tilting of the samples. The lack of area-based information of the surface and the interaction of the touch probe with the samples makes a contactless measurement method desirable [Leach 2011].

For traditional optical systems similar solutions can be found, but the limitations are also given by geometries, which are not possible to reach due to light/shade influence, steep flanks or parts which are too big to be manipulated with an additional axis system [Schuth et al. 2017].
A new approach to this complex measurement task is a metrology platform for industrial measurements that uses a unique combination of collaborative robots, a fast 3D sensor based on Focus-Variation [Lankmair et al 2018], and a software for automation of complex 3D measurements tasks.

In contrast to conventional industrial robots, collaborative robots have the advantage that they can be easily moved by a human operator, so that no time-consuming and complex programming of measurement positions is necessary. Additionally, such robots immediately stop when they are exposed to an external force, such as a collision with a human operator, so that no severe injuries of human operators can happen [ISO 15066 2016]. This measurement setup enables new possibilities where human interactions and the measurements need to happen in the same environment.

This robot setup offers the possibility to position the sensor head in different angels, to measure the sample from different directions and create out of these multiple datasets a single high-resolution $3 \mathrm{D}$ dataset to perform the needed data analysis.

With this measurement design the limitations regarding the accessibility of complex geometries are widened up to offer the possibility to use optical metrology systems for geometries which were previously impossible to measure due to an unfavourable, for optical measurement systems, design of certain features of the part [Leach 2014].

This paper introduces a method to use a custom measurement setup to create a high-resolution $3 \mathrm{D}$ dataset 
which is created from different measurements using the tool centre point (TCP) of a collaborative robot and a standard Focus-Variation Sensor. The comparison to normal FocusVariation systems and to a certified standard should give an overview about the capabilities and options of this setup.

\section{COLLABORATIVE ROBOT WITH A FOCUS- VARIATION SENSOR}

\subsection{D Measurement using Focus-Variation}

The measurements are taken with an R25 Sensor by Alicona, which is based on the technology Focus-Variation. It consists of an optical unit with small depth of focus to be able to scan the specimen vertically with the integrated motorized Z-axis. During the measurement process 3D data together with colour information is continuously collected. The small depth of focus of the optics leads to a very small area, which is displayed sharply at the time of a data acquisition workflow. The algorithm analyses the sharp pixels and creates a high-resolution dataset of the surface containing the colour information and the $Z$ values for each measurement point (see Fig. 1).

The sensor has been specially designed for the use in production environments in order to directly mount it on a robot arm or integrate into a manufacturing machine. Together with the robust technology Focus-Variation this solution offers the possibility to measure surface and form characteristics in a very high resolution down to submicrometres in an unfriendly environment.

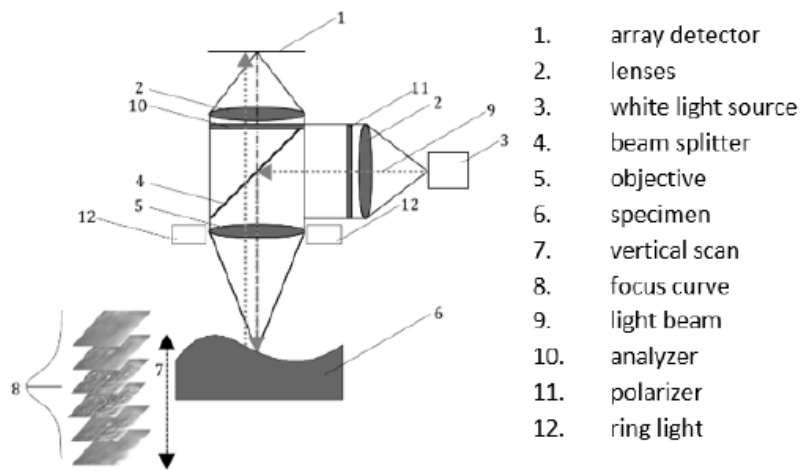

Fig. 1: Schema diagram of a measurement device based on Focus-Variation [Schuth et al. 2017].

\subsection{Hardware setup and preparations}

The used test setup consists of a solid steel frame, a collaborative six-axis robot and the high-resolution FocusVariation sensor with ring light illumination, the IFSensorR25, (see Fig. 2).

After evaluating different collaborative six-axis robots the product UR10 manufactured by Universal Robots was selected due to different parameters like stability, repeatability, stiffness, easy manipulation, possibilities of integration, flexible soft- and hardware interfaces and an overall good usability. The repeatability is less than $\pm 0,1 \mathrm{~mm}$ which is precise enough for the measurements [Gaspar et al 2018]. The payload of $10 \mathrm{~kg}$ is sufficient to move the parts of the measurement system like the sensor head, cables and joysticks.

The used objective in this test setup was a 10xAX objective with a vertical resolution of $130 \mathrm{~nm}$ and a working distance of $33,5 \mathrm{~mm}$.

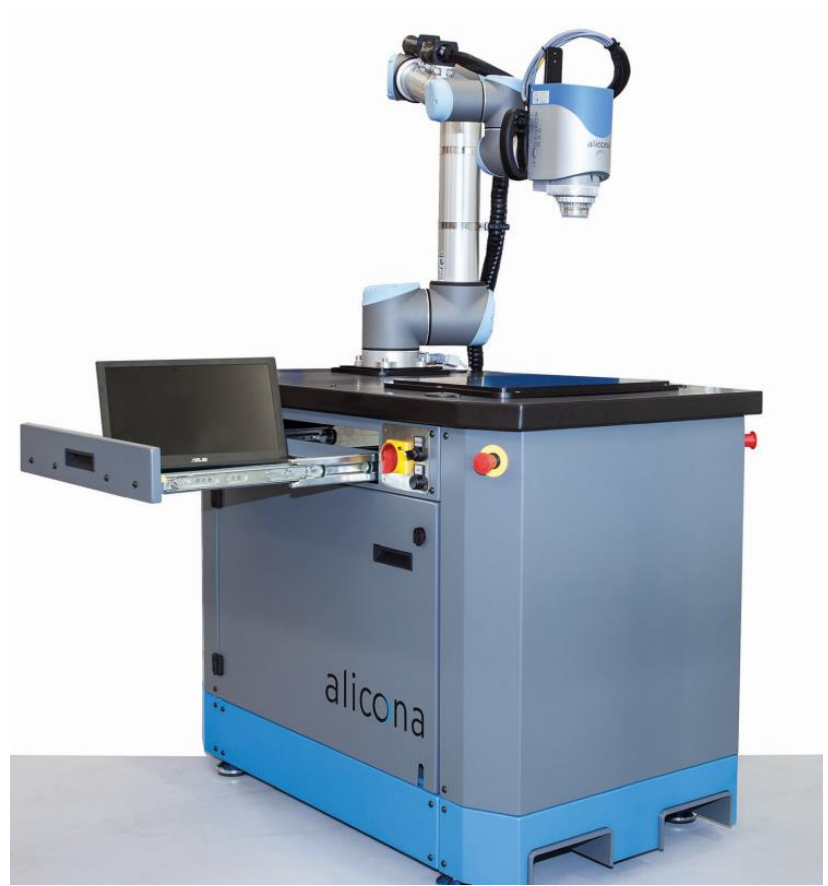

Fig. 2: The used hardware setup.

\subsection{The used artefact}

The used tool for the tests was an artefact, the IFVerificationTool (see Fig. 3), which was calibrated by the PTB. This artefact has different geometric features like step heights, cylinders and angles to be able to perform tests with measurement systems (see Tab. 1) and evaluate the performance of the 3D data acquisitions compared to the values certified by the PTB [GUM 1993].

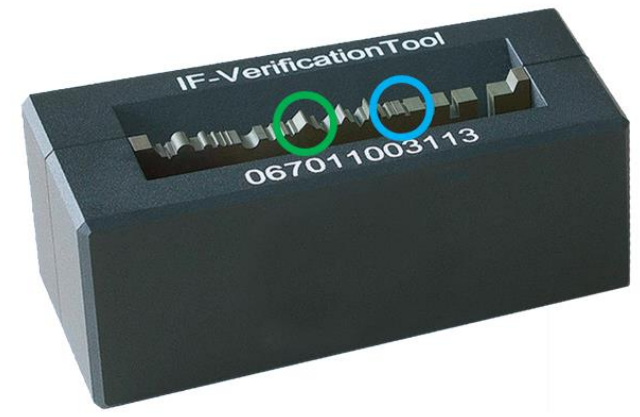

Fig. 3: A similar tool to the used standard (calibrated by the PTB); the measured features are a $90^{\circ}$ angle (circled in green) and a 500 $\mu \mathrm{m}$ step height (circled in blue).

Tab. 1: Technical specifications of the calibration artefact.

\begin{tabular}{|l|l|}
\hline Description & Values \\
\hline Calibrated Angle & $89,97^{\circ}$ \\
\hline Uncertainty for angles & $\mathrm{U}=0,1^{\circ} \mathrm{k}=2$ \\
\hline Calibrated step height & $0,5004 \mathrm{~mm}$ \\
\hline Uncertainty for step height & $\mathrm{U}=0,5 \mu \mathrm{m} \mathrm{k}=2$ \\
\hline $\begin{array}{l}\text { Coefficient of thermal } \\
\text { expansion }\end{array}$ & $(4,5 \pm 0,5) 10^{-6} \mathrm{~K}^{-1}$ \\
\hline $\begin{array}{l}\text { Calibration laboratory } \\
\text { PTB } \\
\text { (Physikalisch-Technische } \\
\text { Bundesanstalt) }\end{array}$ \\
\hline
\end{tabular}




\subsection{Preparations to use the coordinate system of the robot for the measurements}

For the further steps of the test setup the position of the $3 D$ datasets in the robot coordinate system is of high importance and the exact mounting of the Focus-Variation sensor and the objective needs to be taught precisely. The focus point of the measurement system and a special teaching probe (see Fig. 4) were used to perform the steps of the teaching process of the TCP orientation as recommended by the robot manufacturer. In this process the used probe is focused on the same point while moving the robot to 4 different positions. After each movement the current position is confirmed and in the end of the process the robot calculates the TCP of the current setup.

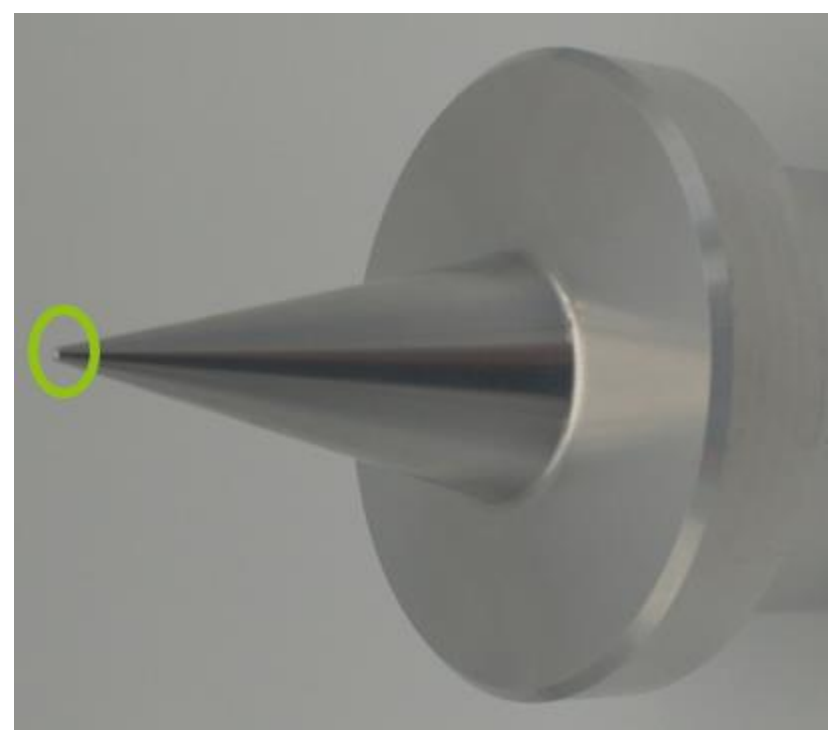

Fig. 4: The probe with the tip for the TCP teaching workflow (circled on green).

This definition of the TCP enables the possibility to know the position of the $3 \mathrm{D}$ data acquisitions in the robot coordinate system. With this information the datasets can be put into a cohere coordinate system even after different movements of the robot [Danzl et al. 2011].

This improves the known process of the Imagefield measurements, which is limited to linear movements (see Fig. 5) to a process without these linear movement restrictions (see Fig. 6).

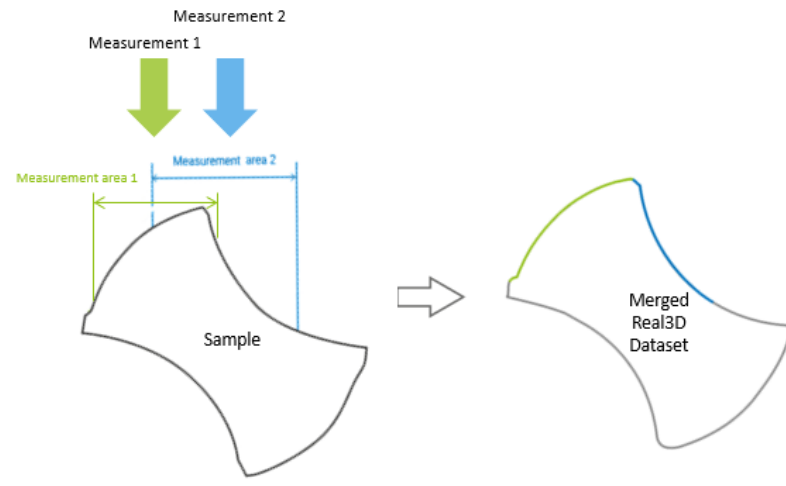

Fig. 5: Schematic view of an ImageField measurement with the linear restriction.

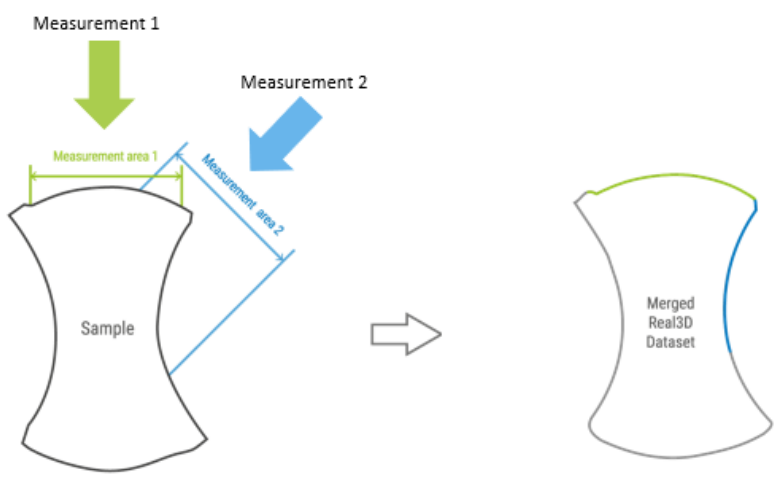

Fig. 6: Schematic view of the new measurement without restrictions.

\subsection{The creation of the datasets}

For the data acquisition (see Fig. 7) an automatic measurement workflow was created and contained the following steps:

1. Waypoint

2. First measurement of the artefact flank

3. Waypoint $X$

4. Second measurement of the artefact flank

5. Waypoint $X$

6. Repeat step 1-5 till the 50 single measurements are finished

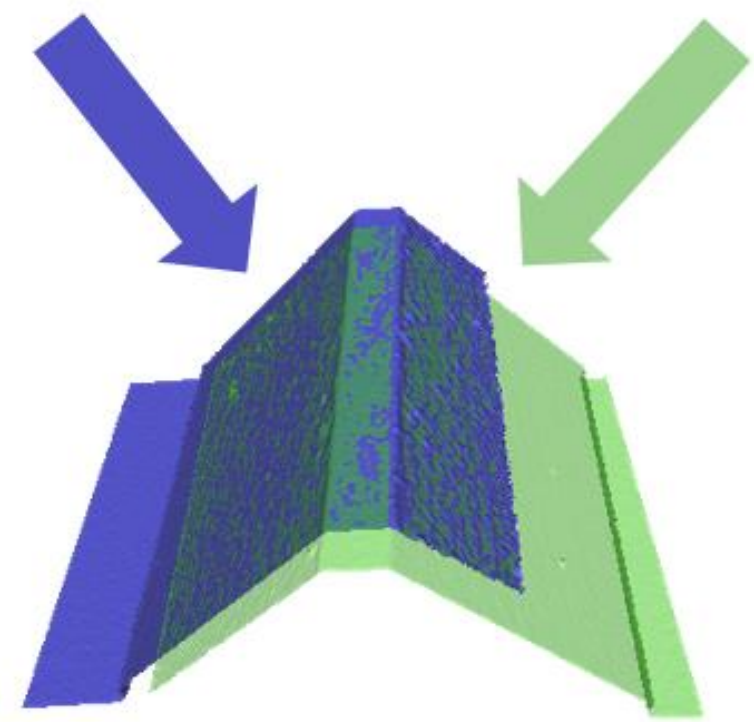

Fig. 7: The angle between the first data acquisition (blue) and the second (green) is roughly $89,8^{\circ}$.

After the acquisition of the single datasets each pair of them is put into the same coordinate system using the TCP coordinates of each single measurement.

The TCP coordinates consist of six different values which determine the position of the $3 \mathrm{D}$ datasets in the base coordinate system of the robot. The first three values describe the $x, y$ and $z$ positions in millimetres in the cartesian coordinate system of the robot base. The last three values characterize the roll, pitch and yaw angles in degrees of the rotation of the robot position denoted with rx, ry and rz respectively [Sciavicco et al. 2012].

These coordinates are directly used to transform the $3 D$ datasets with the help of a 3D analysis software. 
With this step the datasets are roughly in one coordinate system. (see Fig. 8). The fine alignment (see Fig. 9) of the datasets is performed by using an Alicona aligning algorithm. With this process the correct position of the two $3 \mathrm{D}$ datasets is guaranteed. After this step the fusion process, where out of the two datasets the final 3D dataset is created, is performed (see Fig. 10). Here each of the overlapping 3D points is merged with its counterpart on the other dataset.

The offset of the datasets are due to the manual teaching process of the measurement positions and the remaining error of the TCP. In the final merged datasets, it is still visible in the offset, but it has no influence on the quality of the datasets.

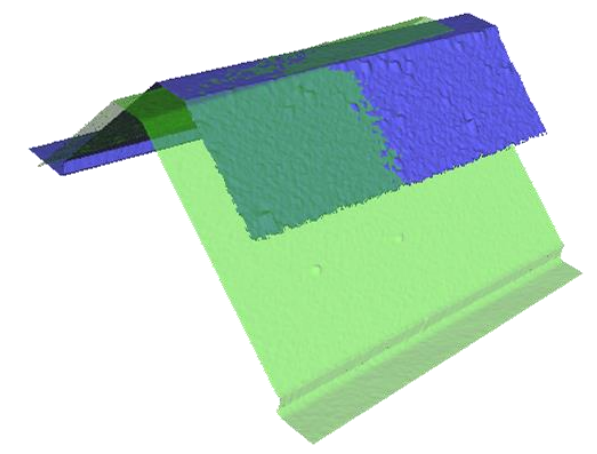

Fig. 8: Two datasets in the same coordinate system after the data acquisition.

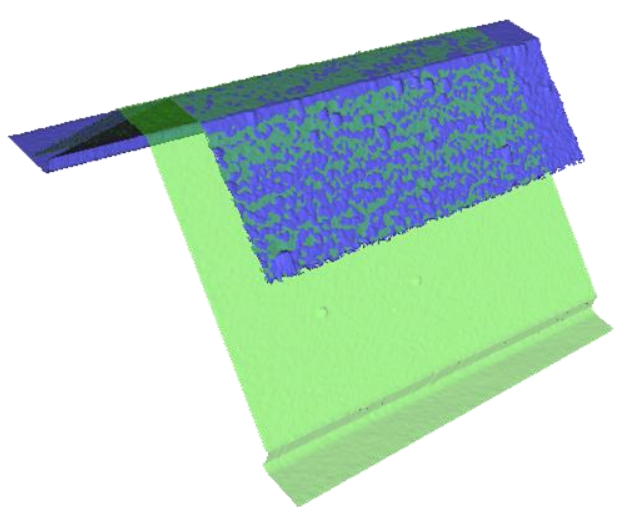

Fig. 9: Two datasets in the same coordinate system after the registration.

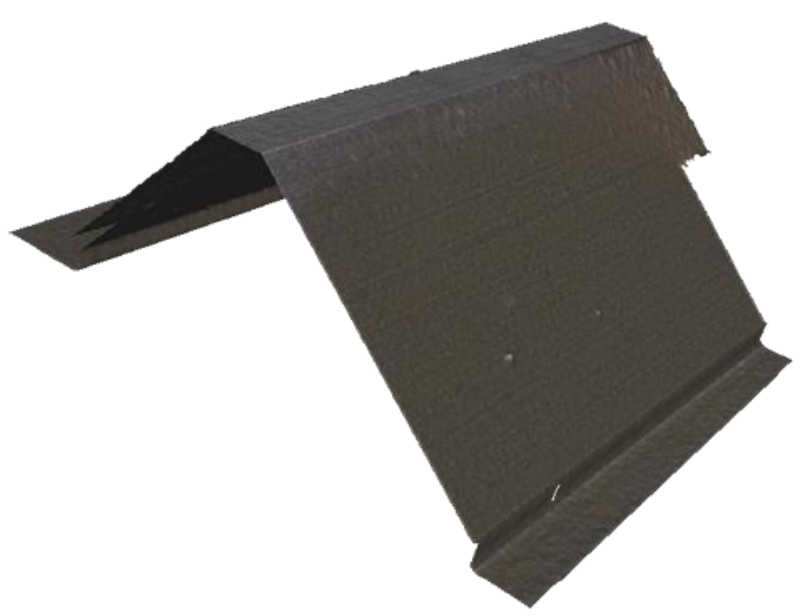

\section{COMPARISON OF THE MEASUREMENT SYSTEMS}

For the evaluation of the capability of the robot measurement setup a standard Focus-Variation system, the InfiniteFocus SL, with a motorized XY stage which uses encoders, was used to check the results of the robot test setup and the resulting datasets. The Focus-Variation sensor and the objective remain the same as used for the robot setup.

For the comparison of the two systems, measurements on both features were performed. Each system made 25 complete measurements on each feature and after that the $3 D$ datasets were analysed. For the evaluation of the datasets two Aliconalnspect analysis were created.

The angle was measured with fitting two planes, using the Gaussian best-fit 3 sigma algorithm, into each flank of each $3 \mathrm{D}$ dataset. Around 40,000 points were used for each plane fit to create these planes and determines the angle between these two planes.

The step height was measured by fitting a plane into the top dataset and calculating the centre of gravity of the bottom surface, both were created by using the Gaussian best-fit 3 sigma algorithm. After that the normal distance of the plane to the centre of gravity was calculated. For the fit of the centre of gravity around $70,0003 \mathrm{D}$ points were used, for the top plane 140,000 .

The results of these analyses were compared with the values which were calibrated by the PTB (see Tab. 1 [ISO 25178-2 2012]).

\subsection{Comparison of the robot and the InfiniteFocus SL measurement for the angle}

The mean values of the results of the 25 measurements, which were performed on both measurement setups, are shown and compared to the PTB certified angle at Tab. 2 [ISO/CD 25178-606: 2015]. Both angles are inside the certified range of the artefact.

Tab. 2: Results of the angel measurement of the InfiniteFocus SL, compared with the calibrated artefact.

\begin{tabular}{|l|l|l|}
\hline Description & Unit & Values \\
\hline Calibrated Angle & $\circ$ & $\begin{array}{l}89,97 \pm \mathrm{U}= \\
0,1^{\circ}(\mathrm{k}=2)\end{array}$ \\
\hline $\begin{array}{l}\text { Measured Angle } \\
\text { InfiniteFocus SL }\end{array}$ & $\circ$ & 89,99 \\
\hline $\begin{array}{l}\text { Measured Angle } \\
\text { Robot }\end{array}$ & $\circ$ & 90,00 \\
\hline
\end{tabular}

The detailed results of each measurement system and of the 25 measurements, compared with the calibrated angel, are shown at Tab. 3. Also depicted are the upper and lower uncertainties as described by the calibration certificate of the PTB [GUM 1993].

Fig. 10: The final merged 3D dataset. 
Tab. 3: Results of the angel measurement of the Robot and the InfiniteFocus SL.

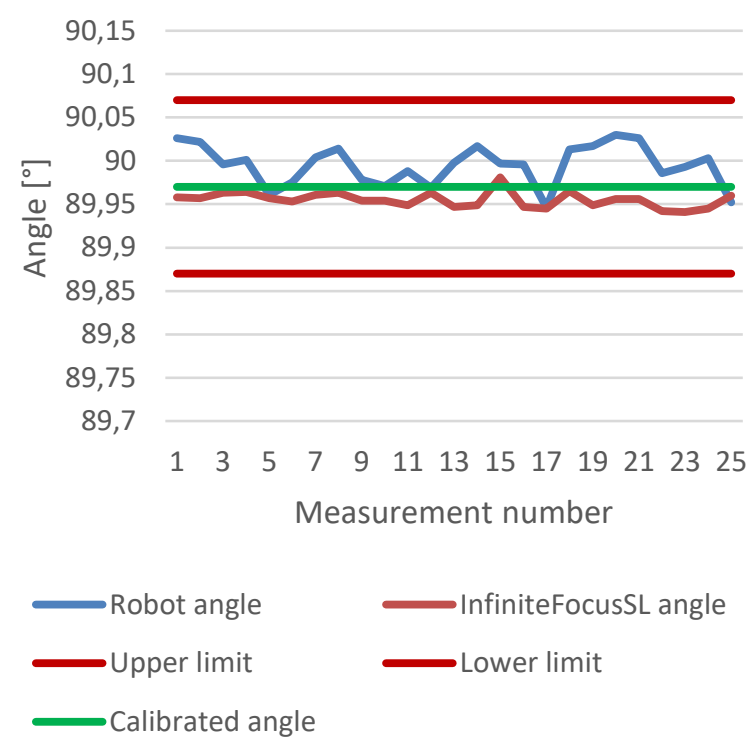

An example of two different datasets in one coordinate system to depict the rough differences is shown at Fig. 11

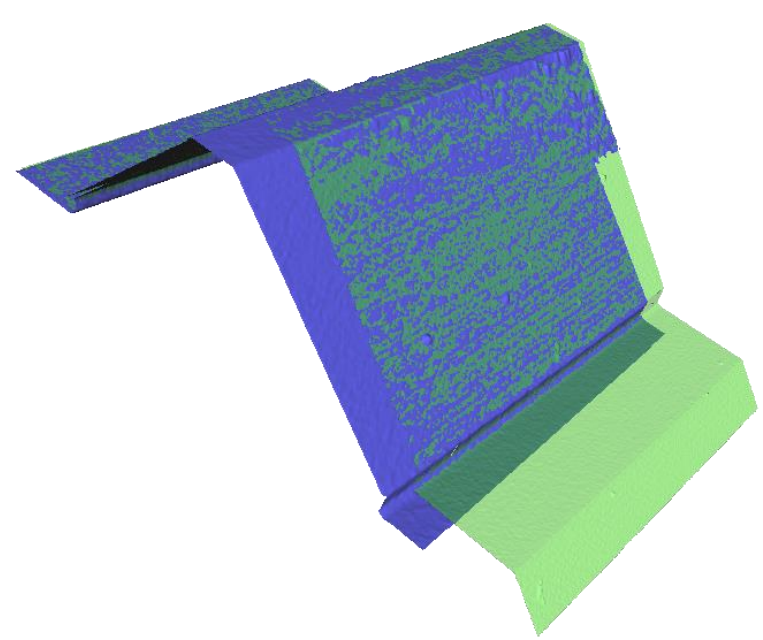

Fig. 11: A measurement made using the Robot (blue) and the InfiniteFocus SL (green) superimposed in one coordinate system.

A difference measurement of two datasets, measured by the robot and the InfiniteFocus SL, using the DifferenceMeasurement of the MeasureSuite to compare and visualize the detailed variation of the data acquisition, was performed. The differences are shown in pseudo colour in a height range of 0 to $10 \mu \mathrm{m}$. The mean deviation of the datapoints used in the difference measurement shows that $98.2 \%$ of the surface are within a $1 \mu \mathrm{m}$ tolerance with a mean deviation of $0.06 \mu \mathrm{m}$ (see Fig. 12). The black parts are differences which occurred due to the different measurement setup and the different dimension of the datasets. These parts have no corresponding data point in the respective dataset.

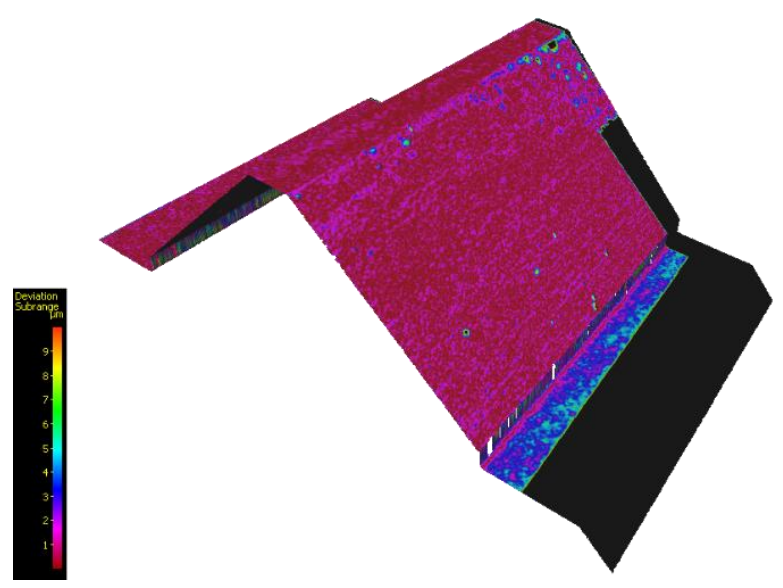

Fig. 12: A difference measurement of the two datasets in pseudo colour (colour range 0-10 $\mu \mathrm{m}$ ).

\subsection{Evaluation of the step heights}

Here also the mean values of the results of the 25 measurements, which were performed on both measurement setups, are shown and compared to the PTB certified step height at Tab. 4 [ISO/CD 25178-606: 2015]. Both step heights are inside the certified range of the artefact.

Tab. 4: Results of the angel measurement of the InfiniteFocus SL, compared with the calibrated artefact.

\begin{tabular}{|l|l|l|}
\hline Description & Unit & Values \\
\hline $\begin{array}{l}\text { Calibrated } \\
\text { height }\end{array}$ & $\mu \mathrm{m}$ & $\begin{array}{l}500,4 \pm \mathrm{U}= \\
0,5(\mathrm{k}=2)\end{array}$ \\
\hline $\begin{array}{l}\text { Measured height } \\
\text { InfiniteFocus SL }\end{array}$ & $\mu \mathrm{m}$ & 500,56 \\
\hline $\begin{array}{l}\text { Measured height } \\
\text { Robot }\end{array}$ & $\mu \mathrm{m}$ & 500,79 \\
\hline
\end{tabular}

The detailed results of each measurement system and of the 25 measurements, compared with the calibrated step height, are shown at Tab. 5. Also depicted are the upper and lower uncertainties as described by the calibration certificate of the PTB [GUM 1993]. 
Tab. 5: Results of the angel measurement of the Robot and the InfiniteFocus SL.

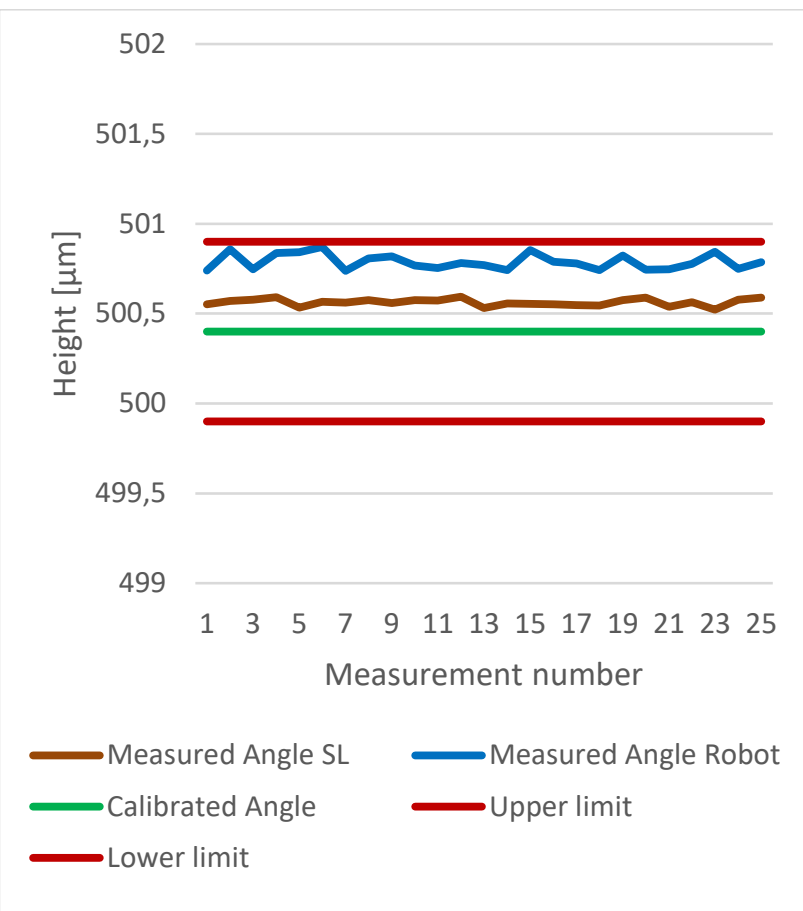

An example of two different datasets in one coordinate system to depict the rough differences is shown in Fig. 13

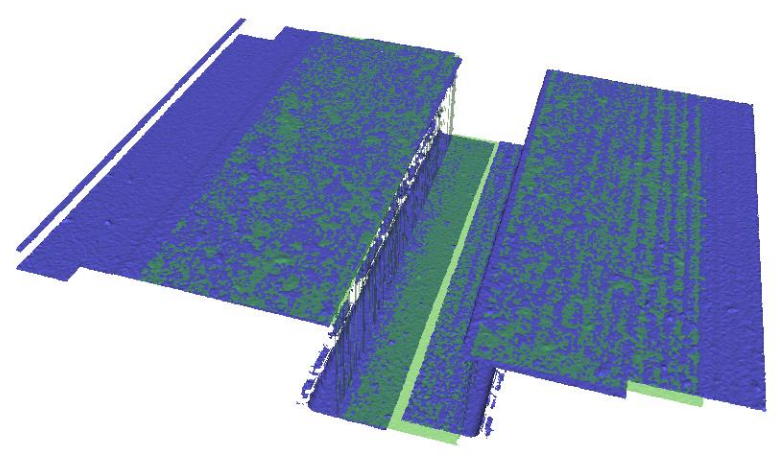

Fig. 13: A measurement made using the Robot (blue) and the InfiniteFocus SL (green) superimposed in one coordinate system.

A difference measurement of two datasets, measured by the robot and the InfiniteFocus SL, using the DifferenceMeasurement of the MeasureSuite to compare and visualize the detailed variation of the data acquisition, was performed. The differences are shown in pseudo colour in a height range of 0 to $10 \mu \mathrm{m}$. The mean deviation of the datapoints used in the difference measurement shows that $94.2 \%$ of the surface are within a $1 \mu \mathrm{m}$ tolerance with a mean deviation of $0.09 \mu \mathrm{m}$ (see Fig. 14). The black parts are differences which occurred due to the different measurement setup and the different dimension of the datasets. These parts have no corresponding data point in the respective dataset.

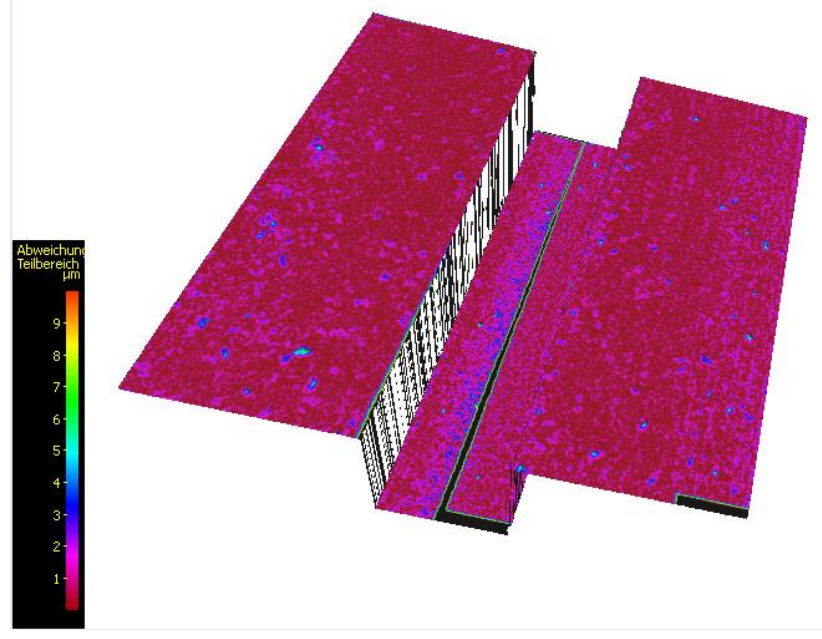

Fig. 14: A difference measurement of the two datasets in pseudo colour (colour range 0-10 $\mu \mathrm{m}$ ).

\section{SUMMARY}

The application of the collaborative robot setup using a high resolution Alicona Focus-Variation sensor is capable of performing high-precision form measurements. It is possible to teach the TCP in such a way that the precision of the measurements is inside the specs of an artefact calibrated by the PTB. This accuracy can be achieved with a standard collaborative robot. The single measurements need a small overlapping area for aligning and merging. The movement workflow of the robot between two different data acquisitions has no influence on the accuracy of the final dataset due to the exact and repeatable positioning of the robot and aligning and merging algorithms of the software. The 3D datasets can be merged together after the complex robot movements and enable measurements around geometric features which were not possible previously with traditional three or five axis optical measurement setups. The evaluation of the resulting datasets shows the high repeatability of this new acquisition method. With this measurement setup there are new possibilities to create high-resolution 3D datasets which were previously impossible to measure due to shading and complex geometric features.

\section{REFERENCES}

[Danzl et al. 2011] Danzl R., Helmli F., Scherer S.. Focus variation - A robust technology for high resolution optical 3D surface metrology, Journal of Mechanical Engineering, 2011;57:245-56

[Gaspar et al 2018] Gaspar T., Bevec R., Ridge B., Ude A. Base Frame Calibration of a Reconfigurable Multi-robot System with Kinestetic Guidance. In: Advances in Service and Industrial Robotics: Proceedings of the 27th International Conference on Robotics in Alpe-Adria Danube Region 2018, Patras, 6-8 June 2018. University of Patras

[GUM 1993] GUM - Guide to the Expression of Uncertainty in Measurement. International Standardization Organization, Geneva, 1993

[ISO 15066 2016] Roboter und Robotikgeräte Kollaborierende Roboter 
[ISO 25178-2 2012] ISO 25178-2 Geometrical product specifications (GPS) - Surface texture: Areal - Part 2: Terms, Definitions and Surface texture Parameters, 2012

[ISO/CD 25178-606: 2015] ISO/CD 25178-606: 2015, Geometrical product specification (GPS) - Surface texture: Areal Part 606: Nominal characteristics of non-contact (focus variation) instruments

[Lankmair et al. 2018] Lankmair, T., Riedl M., Schreink M., Danzl R., 2. VDI-Fachtagung Multisensorik in der Fertigungsmesstechnik 2018, Frankenthal, June 2018, 235-241. ISBN 9783180923260

[Leach 2014] Leach R.K.: Fundamental Principles of Engineering Nanometrology. 2nd ed., Elsevier, 2014

[Leach 2011] R.K. Leach: Optical Measurement of Surface Topography. Berlin, Springer-Verlag, 2011

[Schuth et al. 2017] Schuth M., Buerakov W. Handbuch Optische Messtechnik: Praktische Anwendungen für Entwicklung, Versuch, Fertigung und Qualitätssicherung; Carl Hanser Verlag GmbH Co KG; 2017

[Sciavicco et al. 2012] Sciavicco L., Siciliano B.: Modelling and Control of Robot Manipulators. Berlin, Springer-Verlag, 2012

[Weckenmann 2012] Weckenmann, A. Koordinatenmesstechnik - Flexible Strategien für funktions- und fertigungsgerechtes Prüfen, 2nd ed. Hanser Verlag 2012 